\title{
A Simple Aggregative Algorithm for Counting Triangulations of Planar Point Sets and Related Problems
}

\author{
Victor Alvarez* $\quad$ Raimund Seidel ${ }^{\dagger}$
}

\begin{abstract}
We give an algorithm that determines the number $\operatorname{tr}(S)$ of straight line triangulations of a set $S$ of $n$ points in the plane in worst case time $O\left(n^{2} 2^{n}\right)$. This is the the first algorithm that is provably faster than enumeration, $\operatorname{since} \operatorname{tr}(S)$ is known to be $\Omega\left(2.43^{n}\right)$ for any set $S$ of $n$ points. Our algorithm requires exponential space.

The algorithm generalizes to counting all triangulations of $S$ that are constrained to contain a given set of edges. It can also be used to compute an optimal triangulation of $S$ (unconstrained or constrained) for a reasonably wide class of optimality criteria (that includes e.g. minimum weight triangulations). Finally, the approach can also be used for the random generation of triangulations of $S$ according to the perfect uniform distribution.

The algorithm has been implement and is substantially faster than existing methods on a variety of inputs.
\end{abstract}

\section{Introduction}

For the purposes of this paper a plane graph on a set $S$ of points in the plane is a set $E$ of straight line segments joining points in $S$ that do not intersect except in common endpoints. We will feel free to switch without much ado between the geometric view of such a graph and the discrete view afforded by the naturally associated abstract graph. Let $\mathcal{P G}(S)$ denote the set of all plane graphs on $S$. The set $\mathcal{P} \mathcal{G}(S)$ has received considerable research attention along with some of its subsets, like the set of all plane perfect matchings (all 1-regular graphs in $\mathcal{P G}(S)$ ), the set of all plane cycle covers (all 2-regular graphs in $\mathcal{P G}(S)$ ), the set of all plane spanning cycles (all connected 2-regular graphs), the set of all plane spanning trees (all connected graphs in $\mathcal{P G}(S)$ with a minimal number of edges), and finally the set of all plane triangulations (all graphs in $\mathcal{P G}(S)$ with a maximal number of edges). We will refer to those different subsets of $\mathcal{P G}(S)$ as classes.

For all these classes exponential lower and upper bounds have been proven for their sizes [4, 24, 13, $3,18,27,26,14,25,23,2]$, i.e. statements of the form "for any planar set $S$ of $n$ points the number of plane perfect matchings is $\Omega\left(c_{1}^{n}\right)$ and $O\left(c_{2}^{n}\right)$." However, typically $c_{1}$ and $c_{2}$ are (too) far apart. In some cases the general lower bounds are trivial: e.g. points in convex position admit only one plane spanning cycle.

There has been some interesting work $[10,9,14,20,26]$ on relating the sizes of those classes: E.g. it is easy to see that the number of plane graphs of $S$ is not more than $2^{3 n}$ times the number of plane

\footnotetext{
${ }^{*}$ Fachrichtung Informatik, Universität des Saarlandes, alvarez@cs.uni-saarland.de

${ }^{\dagger}$ Fachrichtung Informatik, Universität des Saarlandes, rseidel@cs.uni-saarland.de
} 
triangulations of $S$. The fact proven by Razen and Welzl [20] that the number plane graphs of $S$ is at least $2^{3 n / 2}$ times the number of plane triangulations of $S$, however, is far from trivial. The same can be said for the result of Sharir, Sheffer, and Welzl that the number of plane perfect matchings of $S$ is at most $O\left(1.1067^{n}\right)$ times the number of plane triangulations of $S$. Still, our understanding of these relationships is quite rudimentary.

A different line of research has addressed the problem of computing the sizes of those various classes of plane graphs for a given point set $S$. Efficient enumeration algorithms are known for all these classes, where "efficient" means polynomial overhead per produced graph. But the interesting problem is to determine the size $N$ of such a class in time that is substantially less than $N$. So far this has been only accomplished for the class of all plane graphs in a recent paper of Razen and Welzl [20], who manage to determine $|\mathcal{P G}(S)|$ in time roughly $O\left(|\mathcal{P G}(S)| / 2^{3 n / 2}\right)$. However, their method does involve enumerating all plane triangulations of $S$.

This paper deals with the class of plane triangulations of $S$. We will refer to it by $\mathcal{T}(S)$ and we will use $\operatorname{tr}(S)=|\mathcal{T}(S)|$. There has been considerable work determining lower and upper bounds for $\operatorname{tr}(S)$. Currently the best bounds known are [23, 26]

$$
\Omega\left(2.43^{n}\right) \leq \operatorname{tr}(S) \leq O\left(30^{n}\right)
$$

where $n=|S|$.

There has also been a fair number of papers on computing $\operatorname{tr}(S)$ given $S$ : The reverse search method of Avis and Fukuda [7] can be applied to enumerate (see [8] for a particularly fast realization), Katoh and Tanigawa [15] consider more general enumeration problems, Aichholzer [1] proposed a divide-and-conquer method base on so-called triangulation paths, Ray and Seidel [19] exploited dynamic programming, and Alvarez, Bringmann, and Ray [6] applied a sweep approach based on triangulation paths and also proposed a method that exploited the onion layer structure of a point set, see [5]. This last approach achieved the so far best worst case running time with a bound of $O\left(3.1414^{n}\right)$. It should be noted that it is unlikely that a polynomial time counting method will be found, since a closely related problem was shown to be NP-hard [16, 22] and even $W$ [2]-hard [5].

In this paper we give an algorithm that determines $\operatorname{tr}(S)$ in worst case time $O\left(n^{2} 2^{n}\right)$ and space $O\left(n 2^{n}\right)$. This running time can well be called substiantially less $\operatorname{than} \operatorname{tr}(S)$, since, as already mentioned, $\operatorname{tr}(S) \geq \Omega\left(2.43^{n}\right)$. The algorithm is suprisingly simple given how long this problem has been studied already. We have implemented the new method and report some experimental results. Our approach can also be used to count all triangulations of $S$ that contain a prescribed set of edges, to find an "optimum" triangulation with respect to certain "decomposable" optimization criteria, or to generate triangulations of $S$ uniformly at random.

\section{The Algorithm}

Let $S=\left\{p_{1}, \ldots, p_{n}\right\}$ be a set of $n$ points in the plane. We assume that $S$ is not contained in a straight line, otherwise there would be no triangulations to count. For the sake of ease of exposition we assume that no three points in $S$ are colinear and no two points lie on a common vertical line. Thus we can assume without loss of generality that the points in $S$ are indexed by increasing $x$ coordinate. A monotone chain for $S$ is a polygonal chain that connects the leftmost point $p_{1}$ and the rightmost point $p_{n}$, contains only points of $S$ as vertices, and intersects every vertical line at most once. 
Let $T$ be some plane triangulation of $S$. From now on we will omit the adjective "plane." A monotone chain in triangulation $T$ is a monotone chain for $S$ all whose segments are edges of the triangulation $T$.

For a monotone chain $C$ in $T$ let $\Delta_{T}(C)$ be the set of triangles of $T$ that lie below $C$. We call a triangle $t$ of $T$ an advance for $C$ if it lies above $C$ and $\Delta_{T}(C) \cup\{t\}=\Delta_{T}\left(C^{\prime}\right)$ for some monotone chain $C^{\prime}$ in $T$. We say that in this case $C$ advances to $C^{\prime}$. Note that there are two different types of advances. Let the advance triangle $t$ be spanned by points $p_{i}, p_{j}, p_{k}$, with $i<j<k$, which also means $p_{i}$ is to the left of $p_{j}$ which in turn is to the left of $p_{k}$. Either $p_{j}$ lies above the segment $\left[p_{i}, p_{k}\right]$. In this case $t$ intersects $C$ in that segment and $C^{\prime}$ is obtained from $C$ by replacing segment $\left[p_{i}, p_{k}\right]$ by the two segments $\left[p_{i}, p_{j}\right],\left[p_{j}, p_{k}\right]$. Thus such an advance increases the length of the chain and includes a new vertex. Otherwise $p_{j}$ lies below $\left[p_{i}, p_{k}\right]$. In this case $t$ intersects $C$ in $\left[p_{i}, p_{j}\right],\left[p_{j}, p_{k}\right]$ and $C^{\prime}$ is obtained from $C$ by replacing those two segments by $\left[p_{i}, p_{k}\right]$. This type of advance decreases the length of the chain and expels vertex $p_{j}$ from the chain.
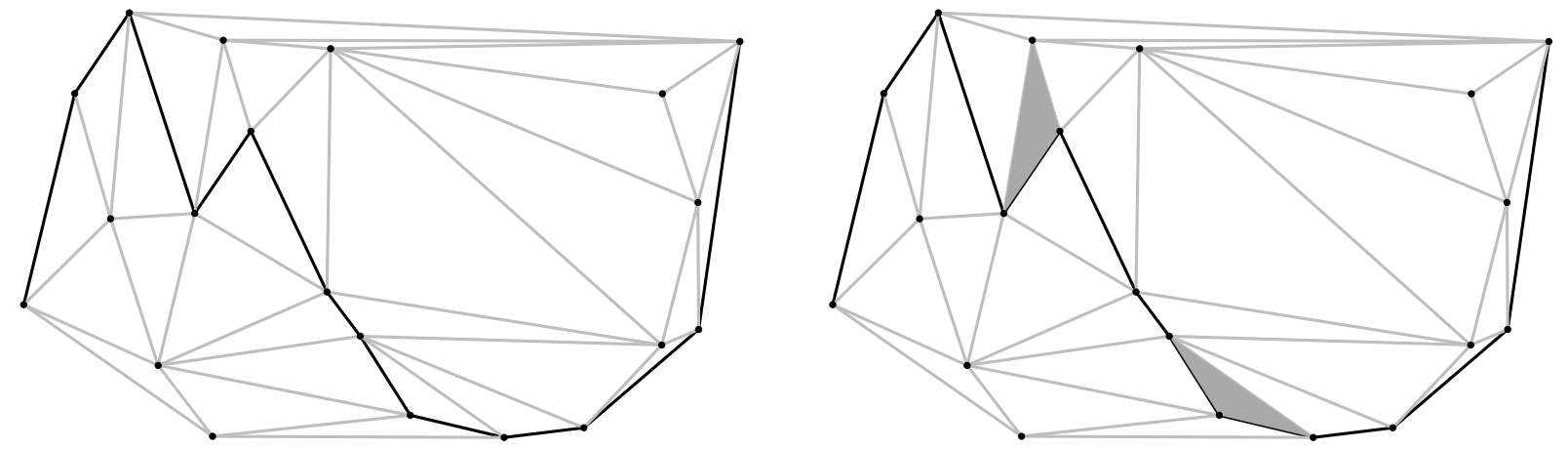

Figure 1: A monotone chain in a triangulation and two possible advances.

The following is folklore:

Lemma 1. Let $T$ be some triangulation of $S$. For any monotone chain $C$ in $T$ there is an advance triangle, unless $C$ consists only of edges of the upper boundary of the convex hull of $S$.

Proof. Let $C$ be a monotone chain in $T$, let $\bar{C}$ be a maximal subchain of $C$ containing no "upper hull edges," and let $p_{h}$ and $p_{\ell}$ the left and right endpoints of $\bar{C}$. Note that for each edge $e=\left[p_{i}, p_{j}\right]$ of $\bar{C}$ there is a unique triangle $t_{e}$ of $T$ that contains $e$ and lies above $\bar{C}$. Let $p$ be the corner of $t_{e}$ that is not on $e$. Assign the orientation left, right, or none to $e$ depending on whether $p$ is to the left of $p_{i}$, to the right of $p_{j}$ or "between" $p_{i}$ and $p_{j}$. If some edge $e$ of $\bar{C}$ has orientation none then $t_{e}$ constitutes an advance triangle. So assume orientation none does not occur. Note that if $e=\left[p_{i}, p_{j}\right]$ is oriented right and the next segment $e^{\prime}=\left[p_{j}, p_{k}\right]$ is oriented left, then $t_{e}=t_{e^{\prime}}$ and this triangle constitutes an advance triangle. But note that such a configuration must occur since the leftmost edge of $\bar{C}$ must be oriented right and the rightmost edge of $\bar{C}$ must be oriented left.

Every monotone chain $C$ in $T$ (except for the topmost one) must have some advance. As a matter of fact it can have several of them. But it has a unique leftmost one, $t$, i.e. no other advance triangle intersects $C$ to the left of $t$. This means that for every triangulation $T$ there is a unique sequence $C_{0}, \ldots, C_{M}$ of monotone chains in $T$, where $C_{0}$ is formed by the lower boundary of the convex hull, $C_{M}$ by the upper boundary of the convex hull, and each $C_{\ell}$ is obtained from $C_{\ell-1}$ by a leftmost 
advance. Here $M$ is the number of triangles in $T$, which must be $2 n-h-2$, where $n=|S|$ and $h$ is the number of points in $S$ that are on the boundary of the convex hull of $S$. We call this unique sequence of chains a leftmost advancing sweep of $T$.

Thus in order to count the number of triangulations of $S$ it suffices to count the number of leftmost advancing sweeps. We will do this by forming a directed acyclic graph $G_{S}$ in which source-sink paths correspond 1-1 with leftmost advancing sweeps. Counting all such source-sink paths can easily be done in time linear in the size of $G_{S}$ by traversing its nodes in topological order.

The nodes of $G_{S}$ will be marked monotone chains for $S$. Such a marked chain is simply a monotone chain for $S$ with one of its edges marked. It can be denoted by the pair $(C, \ell)$, where $C$ is a monotone chain and $\ell$ is an integer indicating that the $\ell$-th edge $e_{\ell}$ of $C$ is marked (counting from left to right).
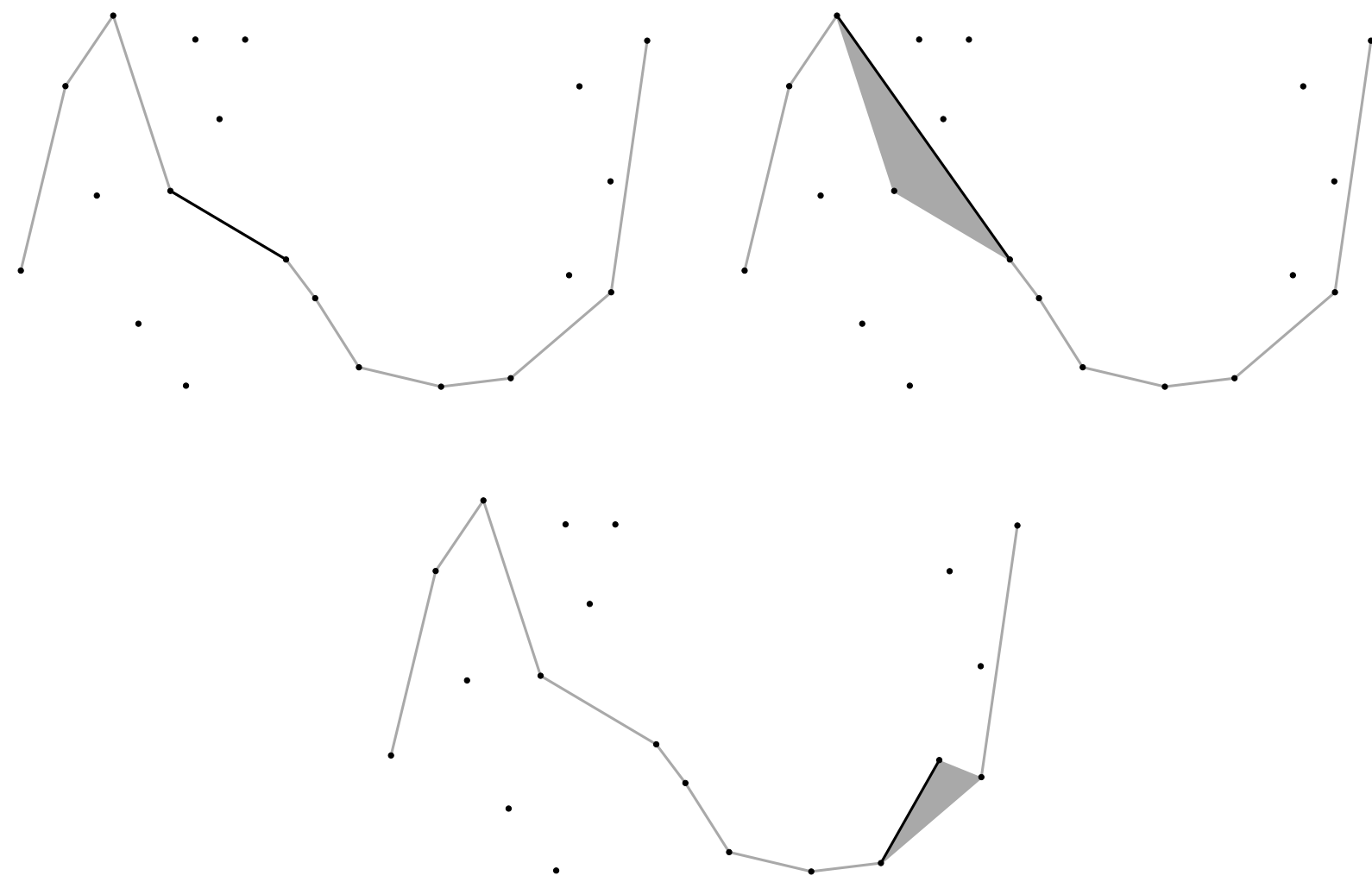

Figure 2: A marked monotone chain for $S$ and two possible successors.

We define a successor relation on such marked monotone chains. Let $(C, \ell)$ be one such marked monotone chain. Let $e_{m}=\left[p_{i}, p_{j}\right]$ with $m \geq \ell$ be the $m$-th edge of $C$. First assume there is some $p_{\mu}$ in $S$ that lies "between" $p_{i}$ and $p_{j}$ (i.e. $i<\mu<j$ ), that lies above $e_{m}$, and for which the triangle spanned by $e_{m}$ and $p_{\mu}$ contains no point of $S$ in its interior. Consider the monotone chain $C^{\prime}$ obtained from $C$ by replacing edge $e_{m}$ by the sequence of two edges $\left[p_{i}, p_{\mu}\right],\left[p_{\mu}, p_{j}\right]$. We define each marked monotone chain $\left(C^{\prime}, m\right)$ obtained this way to be a successor of $(C, \ell)$.

Next assume that chain $C$ contains two consecutive edges $e_{m-1}, e_{m}$ with $m \geq \ell$ so that the triangle spanned by those two edges lies above $C$ and contains no points of $S$ in its interior. Let $p_{i}, p_{j}, p_{k}$ be the three endpoints of the two segments in left to right order, and consider the monotone chain $C^{\prime}$ obtained from $C$ by replacing $e_{m-1}, e_{m}$ by edge $\left[p_{i}, p_{k}\right]$. We define each marked monotone chain 
$\left(C^{\prime}, m-1\right)$ obtained this way to be a successor of $(C, \ell)$.

Our DAG $G_{S}$ contains an edge from $(C, \ell)$ to $\left(C^{\prime}, m\right)$ iff $\left(C^{\prime}, m\right)$ is a successor of $(C, \ell)$.

Let $B$ be the bottom-most monotone chain for $S$, i.e. $B$ contains the sequence of edges on the lower boundary of the convex hull of $S$. Analogously let $U$ be the top-most monotone chain for $S$, i.e. the sequence of edges of the upper boundary of the convex hull of $S$. Let $C$ be some monotone chain for $S$. The following can easily be proven by induction:

Claim 2. There is a 1-1 correspondence between the directed paths in $G_{S}$ from $(B, 1)$ to $(C, k)$ and the triangulations of the area $A$ sandwiched between chains $B$ and $C$ whose leftmost advancing sweep has as last advance a triangle $t$ whose leftmost upper boundary edge is the $k$-th edge of $C$.

The triangulation of $A$ must of course include also all points of $S$ as vertices that lie between $B$ and $C$. Note that for a given chain $C$ all such triangulations have the same number of triangles. We refer to this number as the level of $C$. The induction for proving this claim is on this level number.

Since for any triangulation of $S$ the leftmost advancing sweep has as last advance a triangle whose upper boundary is part of the top-most chain $U$ we get that every path in $G_{S}$ from $(B, 1)$ to $(U, k)$ for some $k$ corresponds to a leftmost advancing sweep of some triangulations of $S$. Adding a "top" vertex $\top$ to $G_{S}$ that has an edge to it from every marked chain $(U, k)$ we get our main theorem:

Theorem 3. The number of triangulations of $S$ is the number of paths in $G_{S}$ from source $(B, 1)$ to $\operatorname{sink} \top$.

The number of those source sink paths can be determined in time $O\left(n^{2} 2^{n}\right)$ and using space $O\left(n 2^{n}\right)$ in the worst case.

Proof. After the preceding discussion we only need to prove the resource bounds.

Determining the number of source-sink paths in a DAG can be achieved by the following algorithm: store a counter with each node that is initialized to 0 ; initialize the counter of the source to 1 ; iterate through the vertices in topological order and for each vertex add its countervalue to the counter of each of its successors.

The time necessary for this algorithm is proportional to the number of edges of the DAG $G_{S}$. Since each monotone chain for $S$ is uniquely identified by a subset of $S$ there are at most $2^{n}$ chains (actually $2^{n-2}$ since $p_{1}$ and $p_{n}$ are always included) and for each chain there are at most $n-1$ possible markings. Thus the number of nodes in our DAG $G_{S}$ is $O\left(n 2^{n}\right)$. Each node can have at most $n$ successors, since each successor either includes a new vertex into the chain or excludes one. Thus the number of edges in $G_{S}$ is $O\left(n^{2} 2^{n}\right)$, which proves the running time bound.

The algorithm does not need to store edges, since given a marked chain, its successors can be computed in $O(n)$ time after polynomial preprocessing. Thus only space for the $O\left(n 2^{n}\right)$ nodes of $G_{S}$ is necessary and the claimed space bound follows.

A short remark on the model of computation: since we use exponential space we have to work with a RAM with linear word size. Therefore it is fair to assume that our counters which can take on exponentially large values can be stored in a single word and can be added in constant time. 


\section{Generalizations}

Our approach admits some easy generalizations. For example, it can be used to determine the number of all triangulations of a set $S$ that are constrained to contain a certain set $R$ of edges. It suffices to observe that such constrained triangulations also have a unique leftmost advancing sweep and to adjust the successor relation for the definition of the DAG $G_{S}$ so that segments that cross edges in $R$ are never considered.

The approach can also be used to compute an "optimal" triangulation of $S$, as long as the function to be optimzed is sufficiently well-behaved. It suffices that the function is defined for any triangulation of any polygonal domain $D$, and for any triangle $t$ the optimum value over all triangulations of $D \cup t$ that contain $t$ can be obtained from the optimum value for $D$ and from $t$. In our algorithm $D$ will be the domain between the bottom chain $B$ and the chain $C$ under consideration and $t$ is the advance triangle sandwiched between $C$ and the successor chain under consideration.

Examples of such well behaved functions are the sum or the maximum of the weights of the triangles (or edges) in a triangulation where the weights are arbitrary numbers assigned to each triangle spanned by three points in $S$. For instance, you could assign to each triangle $t$ the weight $\mid \operatorname{area}(t)-\mu) \mid$, where $\mu$ is the area of the convex hull of $S$ divided by $M$, and $M$ is the number of triangles in any triangulation of $S$, i.e. $M=2 n-h-2$, with $n=|S|$ and $h$ the number of points in $S$ that are on the boundary of the convex hull of $S$. The triangulation that minimizes the sum or the maximum of these weights then consists of triangles whose areas deviate from the mean minimally.

Of course such optimization problems can also be solved for constrained triangulations.

Finally, our approach yields a method for generating a random triangulation of $S$ truly uniformly, unbiased. More precisely, we can preprocess a given point set in time $O\left(n^{2} 2^{n}\right)$ to produce a data structure of size $O\left(n^{2} 2^{n}\right)$ from which we can then generate triangulations of $S$ truly uniformly at random at cost $O\left(n^{2}\right)$ per triangulation.

In spite of the large preprocessing cost this can be quite useful. For instance, we have used this approach to estimate the proportion of the triangulations of $S$ that are regular [12, page 55] by taking sufficiently many random samples and testing each sample triangulation for regularity (which just amounts to solving a linear program). This gave us strong empirical evidence that the proportion of regular triangulations of $n$ points, of which $k$ are extreme, goes to 0 with increasing $n$, when $k$ stays fixed. Details will be described elsewhere.

Here is the random generation method: First we compute the graph $G_{S}$ along with the counter values for each node. We remove all nodes that are not reachable from the source vertex $(B, 1)$ or from which you cannot reach sink vertex $T$.

We choose a random triangulation by constructing a random path: choose a random number $r$ between 1 and $\operatorname{tr}(S)$ and construct the $r$-th source-sink path in reverse order as follows: Starting with $v=\top$ you proceed until $v=(B, 1)$ doing the following: consider the predecessors of $v$ in a canonical order and subtract their counts from $r$ as long as $r$ would stay positive; choose that last predecessor as new $v$. It is an easy exercise to see that each source-sink path is chosen this way with equal probability and hence each triangulation is chosen with equal probability. 


\section{Counting other crossing-free structures}

The main new ingredient in our method is the reduction to counting source-sink paths in a directed acyclic graph. Wettstein and Welzl [28] have managed to discover similar such reductions for counting or enumerating various other crossing free structures on a planar point set, such as perfect matchings, spanning cycles, spanning trees, or all plane graphs.

\section{Dead Ends}

We were hoping that our approach could be adapted to determine the number of (pointed) pseudotriangulations of a point set $S$. So far this has been in vain. The main reason is that Lemma 1 may fail in this context.

Even more importantly, we were hoping that our approach could lead to an improved upper bound for the number of triangulations that every point set can have. The hope was that a combinatorial description of a leftmost advancing sweep would suffice to specify the actual geometric sweep. This combinatorial description would be something of the form: advance chain $C$ by replacing edges $e_{k}, e_{k+1}$ by a single edge, then advance replacing edge $e_{m}=\left[p_{i}, p_{j}\right]$ by the two edges $\left[p_{i}, p_{\lambda}\right],\left[p_{\lambda}, p_{j}\right]$ (without specifying $p_{\lambda}$ ), and so on. If this were possible, then a $27^{n}$ upper bound for the number of triangulations of $S$ would follow, since it is relatively easy to show via a labelling argument that there are at most $3^{3 n}$ "combinatorial leftmost advancing sweeps."

However, there is an old example of Günter Rote [21] that shows that there are point sets that admit two different triangulations that are isomorphic even if the edges are all oriented left to right. Figure 3 shows such a point set. But it is easy to see that two such isomorphic triangulations must have leftmost advancing sweeps that have identical combinatorial descriptions, and thus the combinatorial description by itself cannot specify a unique triangulation.
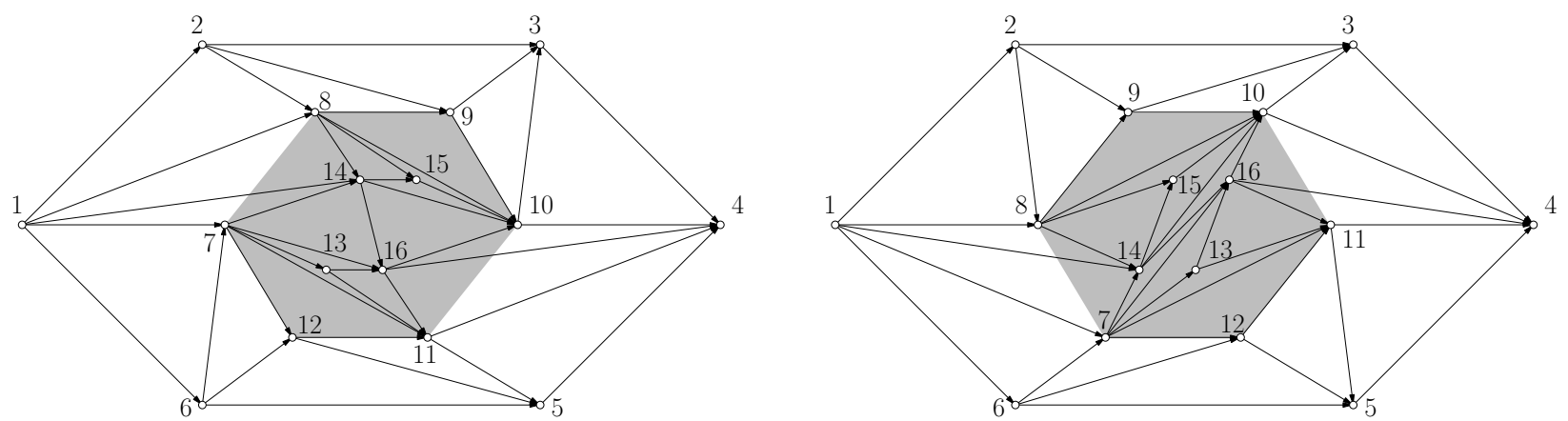

Figure 3: A point set with two different triangulations that are isomorphic even if all edges are oriented left to right.

\section{Implementation Issues}

Our approach is space-intensive. Thus saving space is of utmost importance.

First of all we never store the entire DAG $G_{S}$. We proceed level by level and only store the nodes of at most two consecutive levels. Recall that the level of a chain is the number of triangles in any triangulation below that chain. Say we have proceeded to level $s$ and we have produced the set $L_{s}$ 
of all marked chains of that level that can be reached from source chain $(B, 1)$. We initialize an empty dictionary for $L_{s+1}$. We iterate over $L_{s}$ and for each marked chain $(C, k)$ we generate its successors and for each successor we insert it into $L_{s+1}$ if it is not there already and we add $(C, k)$ 's counter value to the counter value of the successor. After this we can delete $(C, k)$.

Note that a marked chain may not have any successors and may form a "dead end" in $G_{S}$. It is of course desirable to avoid such dead ends as much as possible. So we need conditions that tell us that a given marked chain $(C, k)$ cannot possibly reach $\top$ in $G_{S}$ so that we can remove $(C, k)$ from further consideration right away. A sufficient condition for dismissal of $(C, k)$ is the following: Among the leftmost $k-1$ edges of $C$ there is one that is only visible by points of $S$ that are on or above $C$ and that are left of the $k$-th edge $e_{k}$. The only obstacle for visibility is the chain $C$. Clearly in such a situation any leftmost advancing sweep would have had to advance on a triangle left of $e_{k}$ before, and hence $(C, k)$ cannot occur in such a sweep.

The entries of the dictionaries $L_{s}$ are of the form (key, value), where key is a bit vector representing $(C, k)$ and value is a possibly large integer. $(C, k)$ can be represented by a bit vector of length $n+\log _{2} n$ with $n$ bits specifiying which points of $S$ are vertices of $C$ and the other bits giving the binary representation of $k$. For all we know value could be an integer as large as $30^{n}$, at least this is the best upper bound currently known. Instead of reserving so much space for counters we can resort to Chinese Remaindering and count modulo some prime $P$, for which we only need a fixed number of bits. By running the algorithm several times for different primes and applying the Chinese Remainder Theorem to the resulting count remainders we can recover the true final count.

The dictionaries $L_{s}$ must allow fast search, insertion, and enumeration. Hashing is a natural choice for the realization of such dictionaries. We have found simple hashing with chaining to be too wasteful in space because of the need for pointers. We finally used cuckoo hashing which is reasonably fast and gets by without pointers.

It is possible to avoid dictionaries alltogether. When processing $L_{k}$ simply forego searching and generate copies of (key, value) pairs. In the end we sort on key and aggregate the values of duplicates to one value. This has proven to be too wasteful in terms of space when run on a single machine. However, this way of proceeding is a typical "map-reduce" step [11]. Thus if we are interested in solving large instances using clusters of computers this may well be the simplest way to proceed. Currently the map-reduce paradigm is mostly used for a single step. Our algorithm would need iterated map-reduce steps.

\section{Experiments}

We have implemented our sweep approach and have compared it to two other algorithms, namely the dynamic programming algorithm of [19] and the sn-paths algorithm of [5]. The latter algorithm is tuned for point sets that have few onion layers, i.e. they can be decomposed into the vertex sets of few nested convex polygons. All algorithms were run on a machine with 128 Gigabytes of main memory.

Tables 1 through 3 give some comparisons of running times and space usage. The first table considers $n$ points chosen uniformly at random in a square, the second table considers point sets that essentially are nested triangles, the last table considers points chosen randomly from $k=3$ concentric circles. In these tables $h$ denotes the number of extreme points of the respective set and 
$k$ denotes the number of its onion layers. The term "Base" denotes the $n$-th root of the number to the left; "\# Sub-problems" in "sweep-algo" denotes the maximum number of marked chains encountered at any level.

The sweep algorithm is particularly superior for random input instances, where it could solve problems with up to 50 points, which was completely out of reach before. As expected, the sn-path algorithm performs extremely well for point sets with few onion layers.

The sweep algorithm performs very poorly if all points are in convex position. Figure 4 gives a brief account of this phenomenon.

We also made preliminary experiments with the sweep algorithm for the constrained version of the problem. Figure 5 illustrates those results with two examples. One has a perfect matching as constraint set, the other one the edeges of the minimum spanning tree. Note that the latter example could of course also be solved using dynamic programming, which would take only $O\left(n^{3}\right)$ time.

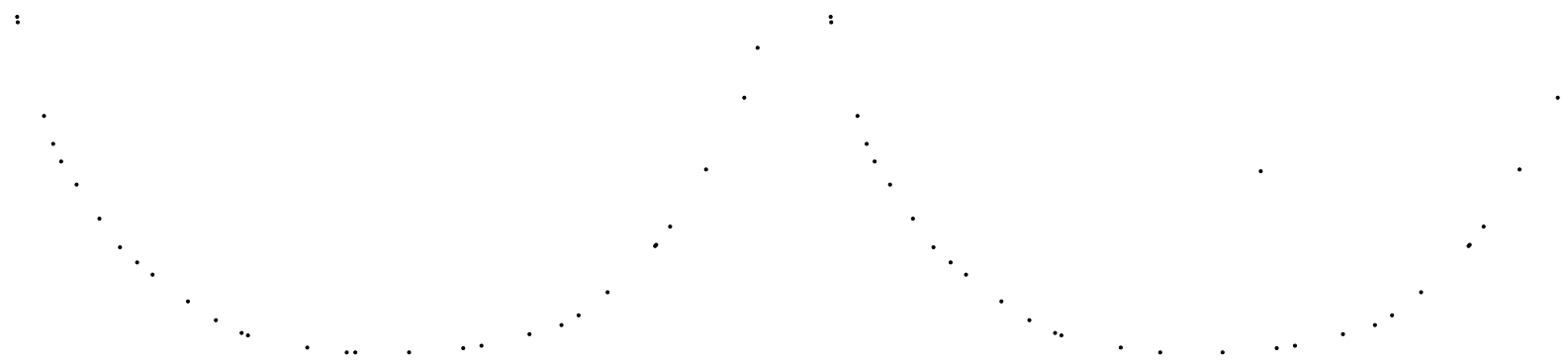

Figure 4: To the left 30 points in convex position which forces the worst-case behavior of the algorithm. It took our program almost 17 minutes and $7050 \mathrm{MB}$ of RAM memory to complete this instance. To the right the same configuration but with one point moved to the interior of the convex hull. This reduces the complexity of the algorithm significantly: almost 9 minutes and 3633 MB of RAM memory.
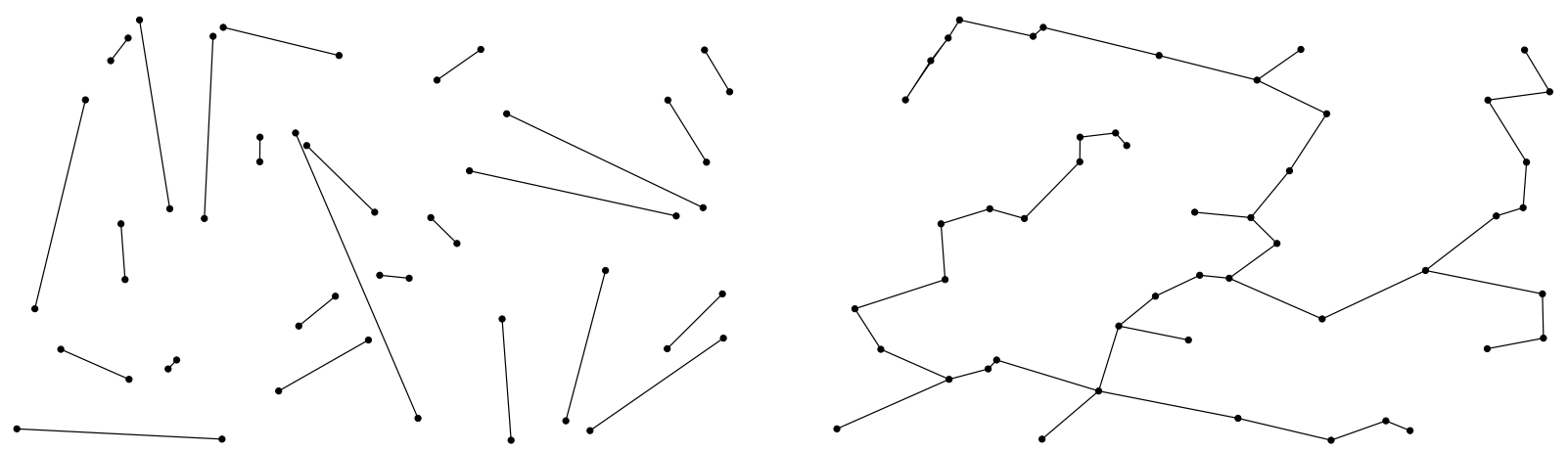

Figure 5: Both shown sets of points are the same. They correspond to the first set of 50 points of Table 1 , having $113071115010855074515830603921337 \approx 4.37^{50}$ triangulations. The number of triangulations containing the shown matching is $1214781483428889112873468 \approx 3.03^{50}$, which constitutes approximately a $1.074 \cdot 10^{-8}$ fraction of the total number of triangulations. The number of triangulations containing the minimum weight spanning tree is $3963846555354291101173440 \approx$ $3.10^{50}$, which is about a $3.505 \cdot 10^{-8}$ fraction of the total number of triangulations. 
Time in hhh:mm:ss.ms

RAM in MB

\# Sub-problems

\begin{tabular}{|c|c|c|c|c|c|c|c|c|c|c|c|c|c|}
\hline$n$ & $k$ & $h$ & \#Triangulations & Base & dyn-prog & sn-paths & sweep-algo & dyn-prog & sn-paths & sweep-algo & dyn-prog & sn-paths & sweep-algo \\
\hline \multirow[t]{2}{*}{30} & 5 & 9 & 29762284427845618 & $\approx 3.54$ & 7.60 & $3: 56.61$ & 5.02 & 130 & 141 & 8 & 854579 & 947262 & 88490 \\
\hline & 6 & 7 & 54648952555202115 & $\approx 3.61$ & 30.69 & $16: 17.12$ & 11.28 & 470 & 535 & 16 & 3150228 & 3590878 & 233038 \\
\hline \multirow[t]{2}{*}{33} & 5 & 11 & 8830953374442248378 & $\approx 3.75$ & 34.80 & $14: 47.26$ & 28.84 & 643 & 394 & 79 & 4245399 & 2554063 & 549137 \\
\hline & 6 & 7 & 23407918365649149382 & $\approx 3.86$ & 15.10 & $1: 10: 34$ & 53.16 & 288 & 1292 & 65 & 1907449 & 8731943 & 723316 \\
\hline \multirow[t]{2}{*}{37} & 5 & 11 & 8317197892568798832050 & $\approx 3.91$ & $3: 35.27$ & 1:15:00 & $8: 44.55$ & 2796 & 1524 & 615 & 18477670 & 9735430 & 3944618 \\
\hline & 5 & 13 & 15347609782987966767248 & $\approx 3.97$ & $15: 23.53$ & $2: 16: 32$ & $9: 21.30$ & 10707 & 1957 & 437 & 70483691 & 12535632 & 7147625 \\
\hline \multirow[t]{2}{*}{40} & 6 & 12 & 1146138971033715203926926 & $\approx 3.99$ & $25: 42.43$ & $13: 29: 43$ & 1:00:49.56 & 18525 & 8889 & 2139 & 121049523 & 56587195 & 35007849 \\
\hline & 7 & 10 & 5050493282169462429012536 & $\approx 4.14$ & $1: 35: 45$ & $46: 49: 41$ & $23: 38.37$ & 54128 & 25533 & 776 & 354717051 & 155716531 & 12694463 \\
\hline \multirow[t]{2}{*}{43} & 6 & 10 & 981403313298259834292202925 & $\approx 4.24$ & $3: 20: 54$ & $107: 48: 48$ & $1: 39: 51.84$ & 116506 & 37407 & 4578 & 752596823 & 239084256 & 49968377 \\
\hline & 7 & 8 & 707769153122173171028848193 & $\approx 4.21$ & & & $3: 43: 21$ & & & 10614 & & & 115902214 \\
\hline \multirow[t]{2}{*}{47} & 7 & 7 & 3620385165725255777788093397554 & $\approx 4.46$ & & & $8: 22: 12$ & & & 11427 & & & 149735651 \\
\hline & 6 & 10 & 1789292526793886024349584939752 & $\approx 4.40$ & & & 15:27:04 & & & 22351 & & & 292928200 \\
\hline \multirow[t]{2}{*}{50} & 6 & 11 & 113071115010855074515830603921337 & $\approx 4.37$ & & & $18: 53: 56$ & & & 30637 & & & 401522629 \\
\hline & 6 & 10 & 147019897942999105259582587551602 & $\approx 4.39$ & & & $26: 41: 00$ & & & 47378 & & & 620962165 \\
\hline
\end{tabular}

Table 1: $n$ random points in a square.

Time in hh:mm:ss.ms

RAM in MB

\# Sub-problems

\begin{tabular}{|c|c|c|c|c|c|c|c|c|c|c|c|c|}
\hline$k$ & $n$ & \#Triangulations & Base & dyn-prog & sn-paths & sweep-algo & dyn-prog & sn-paths & sweep-algo & dyn-prog & sn-paths & sweep-algo \\
\hline \multirow{2}{*}{10} & 28 & 134806114688321888 & $\approx 4.09$ & $1: 04.33$ & $39: 29: 17$ & 33.36 & 1130 & 56197 & 43 & 8015023 & 347448787 & 440822 \\
\hline & 28 & 259051751512786147 & $\approx 4.18$ & 58.55 & $32: 31: 58$ & 13.44 & 903 & 41745 & 21 & 6303203 & 266661064 & 209627 \\
\hline \multirow[t]{2}{*}{11} & 33 & 1360909298406546057232 & $\approx 4.36$ & $33: 23.12$ & & $13: 07.23$ & 26618 & & 474 & 181487896 & & 6186341 \\
\hline & 33 & 1862373658387735722566 & $\approx 4.41$ & $1: 22: 42$ & & $45: 24.99$ & 52344 & & 1866 & 353976704 & & 15271920 \\
\hline \multirow[t]{2}{*}{12} & 35 & 68231356546945667957547 & $\approx 4.49$ & $1: 56: 47$ & & $1: 20: 25.55$ & 78356 & & 2758 & 528028609 & & 30094238 \\
\hline & 34 & 4657839362065190027715 & $\approx 4.33$ & $1: 45: 20$ & & $2: 35: 44.46$ & 73150 & & 4541 & 495166380 & & 59490749 \\
\hline \multirow[t]{2}{*}{13} & 37 & 1075218822593378348459037 & $\approx 4.46$ & & & 10:59:33 & & & 22268 & & & 243201755 \\
\hline & 37 & 1374291968080852706936837 & $\approx 4.49$ & & & $16: 11: 21$ & & & 31168 & & & 408493620 \\
\hline
\end{tabular}

Table 2: $n$ random points having $k=\left\lceil\frac{n}{3}\right\rceil$ onion layers.

Time in hh:mm:ss.ms

RAM in MB

\# Sub-problems

\begin{tabular}{|c|c|c|c|c|c|c|c|c|c|c|c|c|}
\hline$n$ & $h$ & \#Triangulations & Base & dyn-prog & sn-paths & sweep-algo & dyn-prog & sn-paths & sweep-algo & dyn-prog & sn-paths & sweep-algo \\
\hline \multirow[t]{2}{*}{30} & 10 & 161014656152655441 & $\approx 3.74$ & 20.18 & 55.77 & 7.18 & 303 & 33 & 25 & 2050514 & 215732 & 139697 \\
\hline & 10 & 312513373686594183 & $\approx 3.82$ & 1:07.72 & 1:09.17 & 16.46 & 1160 & 38 & 34 & 7879754 & 246657 & 344201 \\
\hline \multirow[t]{2}{*}{33} & 11 & 32155601714553665796 & $\approx 3.90$ & $3: 50.96$ & $2: 19.19$ & $3: 02.25$ & 2762 & 62 & 293 & 18992928 & 405580 & 2178760 \\
\hline & 11 & 68598010833407738067 & $\approx 3.99$ & 58.43 & $2: 30.94$ & $2: 21.51$ & 857 & 62 & 265 & 5812991 & 410357 & 1725109 \\
\hline \multirow[t]{2}{*}{37} & 12 & 9334947679230323509429 & $\approx 3.92$ & $1: 53.60$ & $3: 43.51$ & $9: 47.13$ & 1521 & 90 & 395 & 10027300 & 575255 & 5144450 \\
\hline & 12 & 31113068813012076443512 & $\approx 4.05$ & $3: 20.41$ & $4: 42.24$ & $11: 19.98$ & 2465 & 97 & 664 & 16250100 & 626274 & 7229246 \\
\hline \multirow[t]{2}{*}{40} & 14 & 2642143054680217856074126 & $\approx 4.07$ & $18: 12.16$ & $8: 10.26$ & $31: 33.81$ & 12557 & 131 & 1601 & 82635240 & 866278 & 17458086 \\
\hline & 15 & 2903778262295075928823011 & $\approx 4.08$ & $7: 01.40$ & 9:50.98 & $1: 02: 46.86$ & 4325 & 149 & 3586 & 28333612 & 982791 & 39139805 \\
\hline \multirow[t]{2}{*}{43} & 14 & 452371697808162396583055656 & $\approx 4.16$ & $1: 34: 48$ & $21: 39.66$ & $1: 50: 7.77$ & 53263 & 243 & 5008 & 347603518 & 1604269 & 54673756 \\
\hline & 14 & 461550214764369881018564051 & $\approx 4.16$ & & $19: 25.53$ & $2: 56: 09.84$ & & 242 & 8612 & & 1591423 & 94039067 \\
\hline \multirow[t]{2}{*}{47} & 16 & 157759710540671985436621922639 & $\approx 4.18$ & & $32: 46.68$ & 10:02:04 & & 363 & 17507 & & 2287764 & 229438083 \\
\hline & 15 & 341037585238678346710372748758 & $\approx 4.24$ & & $39: 33.42$ & $5: 32: 04$ & & 420 & 8971 & & 2720786 & 117544183 \\
\hline \multirow[t]{2}{*}{50} & 16 & 54782168649020627430413001433261 & $\approx 4.31$ & & $1: 06: 53$ & $57: 16: 32$ & & 606 & 71609 & & 3631525 & 938562001 \\
\hline & 16 & 158997592723683977758501079915910 & $\approx 4.40$ & & $1: 07: 19$ & 30:07:44 & & 553 & 46872 & & 3998798 & 614328639 \\
\hline
\end{tabular}

Table 3: $n$ random points on three concentric circles, each having $\approx \frac{n}{3}$ points. 


\section{Acknowledgements}

Special thanks to Günter Rote for letting us use his original eps-file for Figure 3, and many thanks to Bernd Finkbeiner for letting us use extensively the large main memory machine of his group.

\section{References}

[1] O. Aichholzer, "The path of a triangulation", in Symposium on Computational Geometry, pp. 14-23, 1999. One citation on page 2 .

[2] O. Aichholzer, F. Aurenhammer, H. Krasser, and B. Speckmann, "Convexity minimizes pseudo-triangulations", Comput. Geom., vol. 28, no. 1, pp. 3-10, 2004. One citation on page 1.

[3] O. Aichholzer, T. Hackl, B. Vogtenhuber, C. Huemer, F. Hurtado, and H. Krasser, "On the number of plane geometric graphs", Graphs and Combinatorics, vol. 23(1), pp. 67-84, 2007. One citation on page 1.

[4] M. Ajtai, V. Chvátal, M. Newborn, and E. Szemerédi, "Crossing-free subgraphs", in Theory and Practice of Combinatorics A collection of articles honoring Anton Kotzig on the occasion of his sixtieth birthday (G. S. Peter L. Hammer, Alexander Rosa and J. Turgeon, eds.), vol. 60 of North-Holland Mathematics Studies, pp. 9 -12, North-Holland, 1982. One citation on page 1.

[5] V. Alvarez, K. Bringmann, R. Curticapean, and S. Ray, "Counting crossing-free structures", in Symposium on Computational Geometry (T. K. Dey and S. Whitesides, eds.), pp. 61-68, ACM, 2012. 3 citations on pages 2 and 8.

[6] V. Alvarez, K. Bringmann, and S. Ray, "A simple sweep line algorithm for counting triangulations and pseudo-triangulations", Submitted, 2012. One citation on page 2.

[7] D. Avis and K. Fukuda, "Reverse search for enumeration", Discrete Applied Mathematics, vol. 65 , no. 1-3, pp. 21-46, 1996. One citation on page 2.

[8] S. Bespamyatnikh, "An efficient algorithm for enumeration of triangulations", Comput. Geom., vol. 23, no. 3, pp. 271-279, 2002. One citation on page 2 .

[9] K. Buchin and A. Schulz, "On the number of spanning trees a planar graph can have," in ESA (M. de Berg and U. Meyer, eds.), vol. 6346 of Lecture Notes in Computer Science, pp. 110-121, Springer, 2010. One citation on page 1.

[10] K. Buchin, C. Knauer, K. Kriegel, A. Schulz, and R. Seidel, "On the number of cycles in planar graphs," in COCOON (G. Lin, ed.), vol. 4598 of Lecture Notes in Computer Science, pp. 97-107, Springer, 2007. One citation on page 1.

[11] J. Dean and S. Ghemawat, "Mapreduce: Simplified data processing on large clusters," in 6th Symposium on Operating System Design and Implementation (OSDI 2004), San Francisco, California, USA, December 6-8, pp. 137-150, USENIX Association, 2004. One citation on page 8. 
[12] J. A. De Loera, J. Rambau, F. Santos, Triangulations: Structures for Algorithms and Applications, Algorithms and Computation in Mathematics, Vol. 25, Springer-Verlag One citation on page 6 .

[13] P. Flajolet and M. Noy, "Analytic combinatorics of non-crossing configurations," Discrete Mathematics, vol. 204, no. 1-3, pp. 203-229, 1999. One citation on page 1.

[14] M. Hoffmann, M. Sharir, A. Sheffer, C. D. Tóth, and E. Welzl, "Counting plane graphs: Flippability and its applications", in WADS (F. Dehne, J. Iacono, and J.-R. Sack, eds.), vol. 6844 of Lecture Notes in Computer Science, pp. 524-535, Springer, 2011. One citation on page 1.

[15] N. Katoh and S.-I. Tanigawa, "Fast Enumeration Algorithms for Non-crossing Geometric Graphs", Discrete \& Computational Geometry, vol. 42, no. 3, pp. 443-468, 2009. One citation on page 2 .

[16] E. L. Lloyd, "On triangulations of a set of points in the plane," in FOCS, pp. 228-240, IEEE Computer Society, 1977. One citation on page 2.

[17] P. McCabe and R. Seidel, "New Lower Bounds for the Number of Straight-Edge Triangulations of a Planar Point Set", in European Workshop on Computational Geometry, 2004. No citations.

[18] A. G. Olaverri, M. Noy, and J. Tejel, "Lower bounds on the number of crossing-free subgraphs of $\mathrm{K}_{\mathrm{n}}$ ", Comput. Geom., vol. 16, no. 4, pp. 211-221, 2000. One citation on page 1.

[19] S. Ray and R. Seidel, "A simple and less slow method for counting triangulations and for related problems", in European Workshop on Computational Geometry, 2004. 2 citations on pages 2 and 8 .

[20] A. Razen and E. Welzl, "Counting plane graphs with exponential speed-up", in Rainbow of Computer Science (C. S. Calude, G. Rozenberg, and A. Salomaa, eds.), vol. 6570 of Lecture Notes in Computer Science, pp. 36-46, Springer, 2011. 3 citations on pages 1 and 2.

[21] G. Rote, Private Communication. One citation on page 7.

[22] A. Schulz, "The existence of a pseudo-triangulation in a given geometric graph," in EuroCG, 2006. One citation on page 2 .

[23] M. Sharir and A. Sheffer, "Counting triangulations of planar point sets", Electr. J. Comb., vol. 18, no. 1, 2011. 2 citations on pages 1 and 2 .

[24] M. Sharir and A. Sheffer, "Counting plane graphs: Cross-graph charging schemes," CoRR, vol. abs/1209.0194, 2012. One citation on page 1.

[25] M. Sharir, A. Sheffer, and E. Welzl, "On degrees in random triangulations of point sets", J. Comb. Theory, Ser. A, vol. 118, no. 7, pp. 1979-1999, 2011. One citation on page 1.

[26] M. Sharir, A. Sheffer, and E. Welzl, "Counting plane graphs: perfect matchings, spanning cycles, and kasteleyn's technique", in Symposium on Computational Geometry (T. K. Dey and S. Whitesides, eds.), pp. 189-198, ACM, 2012. 3 citations on pages 1 and 2.

[27] M. Sharir and E. Welzl, "On the number of crossing-free matchings, cycles, and partitions", SIAM J. Comput., vol. 36, no. 3, pp. 695-720, 2006. One citation on page 1. 
[28] M. Wettstein, "Algorithms for counting crossing-free configurations", M.Sc. Thesis, Dept. of Computer Science, ETH Zürich, 2013. One citation on page 7. 